\title{
Is the Low-Grade Squamous Intraepithelial Lesion/Atypical Squamous Cells Cannot Exclude High-Grade Squamous Intraepithelial Lesion Category Associated with Cervical Intraepithelial Neoplasia 2?
}

\author{
Handan Çetiner ${ }^{a} \quad$ Gözde Kır $^{b} \quad$ Ecmel Kaygusuz $^{\mathrm{a}}$ Yeşim Sağlıcan ${ }^{c}$ Canan Kabaca ${ }^{d}$ \\ a Pathology Department, Zeynep Kamil Hospital, b Pathology Department, Umraniye Hospital, cPathology Department, \\ Acıbadem Hospital, and d Gynecological Oncology Department, Zeynep Kamil Hospital, Istanbul, Turkey
}

\section{Key Words}

Cervical intraepithelial neoplasia - Low-grade squamous intraepithelial lesion cannot exclude high-grade squamous intraepithelial lesion · Bethesda system · Cervical cytology

\begin{abstract}
Objective: A number of cervical smears may exhibit unequivocal low-grade squamous intraepithelial lesions (LSIL) in association with atypical cells cytomorphologically suspicious, but not sufficient to be interpreted as high-grade squamous intraepithelial lesions (HSIL). These lesions are presently called LSIL, atypical squamous cells cannot exclude HSIL (LSIL/ASC-H). Previous studies have shown that LSIL/ASC-H and ASC-H are both equivocal for HSIL and have a high risk of underlying HSIL. However, in researching the literature only two studies were found which rendered the results as cervical intraepithelial neoplasia (CIN) 2 and CIN3 separately. The purpose of this study was to compare the distribution of biopsy results for CIN2 and CIN3 in patients with ASC-H, HSIL, and LSIL/ASC-H. Study Design: Between January 2005 and December 2011, cervicovaginal smears $(98,594)$ with a diagnosis of ASC-H, LSIL, LSIL/ASC-H, or HSIL were re-evaluated to determine the prevalence of future lesion development. Results: A total of 252 patients who had histologic follow-up within a year were selected. Among
\end{abstract}

these, LSIL/ASC-H (31.7\%) had the highest prevalence of CIN2 between LSIL (9.3\%), ASC-H (10\%), and HSIL (16\%). All differences were statistically significant. Conclusion: Because of the high predictive value of CIN2, LSIL/ASC-H may have further importance, especially in women of different age groups.

(c) 2013 S. Karger AG, Base

\section{Introduction}

On an average day in a pathology laboratory, cytopathologists see slides of cervical smears which are in equivocal categories of atypical squamous cells of undetermined significance (ASC-US) and atypical squamous cells cannot exclude high-grade squamous intraepithelial lesions (ASC-H) which represent equivocal low-grade squamous intraepithelial lesions (LSIL) and equivocal high-grade squamous intraepithelial lesions (HSIL), respectively, as included in the Bethesda system (TBS) 2001 [1]. There is also a subset of cases which cannot easily be diagnosed with TBS because they are a separate category entirely. In those cases, there is the challenge of overcalling LSIL or undercalling HSIL. These cases typically contain numerous cells of LSIL and only a few cells with features suggesting HSIL. There is a great deal of published

\section{KARGER}

E-Mail karger@karger.com

www.karger.com/acy
(C) 2013 S. Karger AG, Basel

0001-5547/13/0576-0581\$38.00/0
Correspondence to: Dr. Handan Çetine

Sirmaperde sok. Altunizade Konutları C Blok Daire: 5

Altunizade, Üsküdar

TR-34662 Istanbul (Turkey)

E-Mail handancetiner@gmail.com 


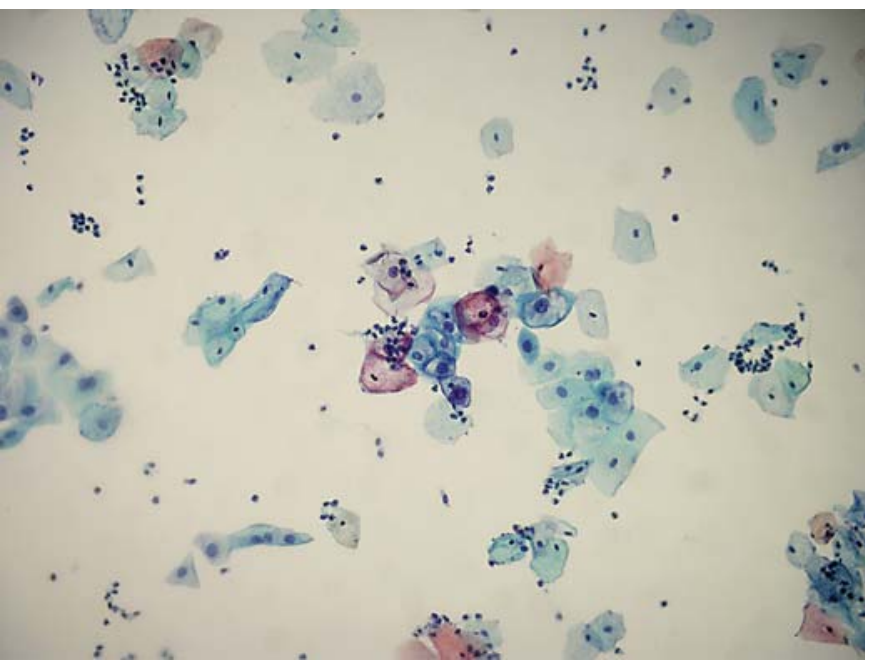

Fig. 1. The background in this smear shows unequivocal LSIL (Pap stain, $\times 100)$.

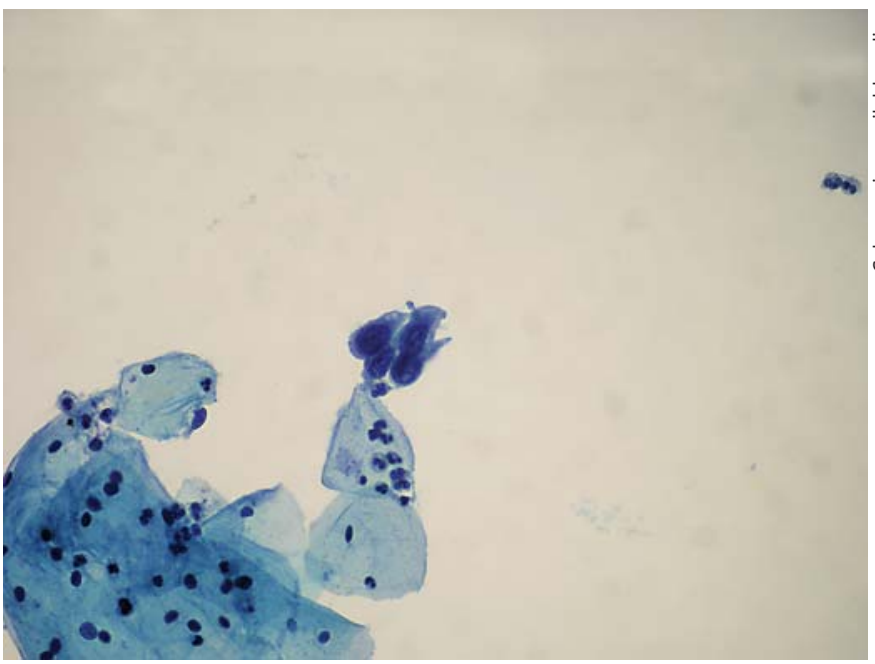

Fig. 2. A little group of ASC-H cells in the same smear (Pap stain, $\times 400)$.

Table 1. Histopathologic outcomes associated with Pap test interpretations of LSIL, ASC-H, LSIL-ASC-H, and HSIL

\begin{tabular}{lllll}
\hline \multirow{2}{*}{$\begin{array}{l}\text { Histologic } \\
\text { diagnosis }\end{array}$} & \multicolumn{4}{l}{ Cytologic interpretation, $\mathrm{n}(\%)$} \\
\cline { 2 - 5 } & $\begin{array}{l}\text { LSIL } \\
(\mathrm{n}=51)\end{array}$ & $\begin{array}{l}\text { ASC-H } \\
(\mathrm{n}=60)\end{array}$ & $\begin{array}{l}\text { LSIL-ASC-H } \\
(\mathrm{n}=60)\end{array}$ & $\begin{array}{l}\text { HSIL } \\
(\mathrm{n}=81)\end{array}$ \\
\hline Benign & $11(21)$ & $21(35)$ & $11(18.3)$ & $16(19.8)$ \\
CIN1 & $33(64.7)$ & $11(18.3)$ & $21(35)$ & $9(11.1)$ \\
CIN2 & $5(9.8)$ & $6(10)$ & $19(31.7)$ & $13(16)$ \\
CIN3 & $5(9.8)$ & $19(31.7)$ & $7(10.6)$ & $33(40.7)$ \\
Carcinoma & $0(0)$ & $3(5)$ & $2(3.3)$ & $10(12.3)$ \\
\hline
\end{tabular}

literature on how well a finding of LSIL/ASC-H cytology predicts HSIL on subsequent histological examination [1-8]. Nearly all of the articles recommend that LSIL/ ASC-H be a separate category in TBS and that a follow-up colposcopy should be performed clinically for all of these patients. The objective of the current study was to determine whether LSIL/ASC-H especially predicts any CIN category, specifically CIN2 and CIN3, and to conclude whether this prediction influences the management of patients.

\section{Materials and Methods}

Cytopathology files from the Pathology Departments of Zeynep Kamil Maternity Hospital and Ümraniye State Hospital containing 98,594 cases collected between January 2005 and December 2011 were database-searched for cervicovaginal smears which had diagnoses of ASC-H, LSIL, LSIL-ASC-H, or HSIL among the conventional Pap smears. Among the 98,594 conventional Pap smears, 333 cases with a diagnosis of ASC-H, LSIL, LSIL-ASC-H, or HSIL were selected. Of these 333 patients, a total of 252 patients having had histologic follow-up within a year were chosen. These Pap smears and biopsies were re-evaluated by 3 pathologists (H.C., E.K., and G.K.) blindly, using a multiheaded microscope. The dysplasia with the highest degree was recorded from the biopsy results in cases that had undergone multiple biopsies. The results were classified under 5 histopathological diagnoses as: benign, CIN1, CIN2, CIN3, or carcinoma.

The diagnosis of LSIL/ASC-H was made when the slides revealed unequivocal LSIL cytologic changes accompanied by ASC$\mathrm{H}$ cells showing cytomorphologic features reported previously [4, 5, 9] (fig. 1, 2). The Number Cruncher Statistical System 2007 and PASS 2008 Statistical Software (USA) programs were used for statistical analysis. Results were compared using the $\chi^{2}$ test. $p<0.05$ was considered statistically significant.

\section{Results}

We studied 98,594 conventional Pap smears from patients aged $16-70$ years, over a 5-year period. Of these, 333 cases were interpreted as abnormal (108 LSIL, 77 LSIL/ASC-H, 61 ASCH, and 87 HSIL). Histologic followups (within a year after the Pap smear) were available for 252 of the $333(75.3 \%)$ cases as follows: 51 LSIL, 60 LSIL/ ASC-H, 60 ASC-H, and 81 HSIL. The biopsy results were grouped as: benign, CIN1, CIN2, CIN3, and carcinoma as shown in table 1.

ASC-H (35\%) had a higher prevalence of negative biopsy results compared to LSIL (21\%), LSIL/ASC-H (18.3\%), and HSIL (19.8\%). LSIL (64.7\%) had higher prevalence of CIN1 than did ASC-H (18.3\%), LSIL/ASC$\mathrm{H}(35 \%)$, and HSIL (11\%). LSIL/ASC-H (31.7\%) had the 
Table 2. Results of the $\chi^{2}$ test for testing differences in cytologic interpretations for each histopathological outcome ( $\mathrm{p}$ values)

\begin{tabular}{lllllll}
\hline & $\begin{array}{l}\text { LSIL vs. } \\
\text { ASC-H }\end{array}$ & $\begin{array}{l}\text { LSIL vs. } \\
\text { LSIL-ASC-H }\end{array}$ & $\begin{array}{l}\text { LSIL vs. } \\
\text { HSIL }\end{array}$ & $\begin{array}{l}\text { ASC-H vs. } \\
\text { LSIL-ASC-H }\end{array}$ & $\begin{array}{l}\text { ASC-H vs. } \\
\text { HSIL }\end{array}$ & $\begin{array}{l}\text { LSIL-ASC-H vs. } \\
\text { HSIL }\end{array}$ \\
\hline Benign & $0.030^{*}$ & 0.119 & 0.801 & $0.039^{*}$ & $0.042^{*}$ & 0.832 \\
CIN1 & $0.001^{* *}$ & $0.002^{* *}$ & $0.001^{* *}$ & $0.039^{*}$ & 0.224 & $0.001^{* *}$ \\
CIN2 & 0.894 & $0.001^{* *}$ & $0.033^{*}$ & $0.003^{* *}$ & 0.298 & $0.029^{*}$ \\
CIN3 & $0.005^{* *}$ & 0.887 & $0.001^{* *}$ & $0.004^{* *}$ & 0.270 & $0.001^{* *}$ \\
Carcinoma & 0.404 & 0.188 & $0.009^{* *}$ & 0.648 & 0.136 & 0.058 \\
\hline
\end{tabular}

* Significant at the $5 \%$ significance level; ${ }^{* *}$ significant at the $1 \%$ significance level.

highest prevalence of CIN2 between LSIL (9.3\%), ASC-H (10\%), and HSIL (16\%) (table 1). Also, HSIL (40.7\%) had a higher prevalence of CIN3 in the biopsy results compared to LSIL (5\%) and LSIL/ASC-H [6, 10]. HSIL (12.3\%) had a higher prevalence of carcinoma compared to LSIL (table 1). All of these differences were statistically significant (table 2).

\section{Discussion}

Aside from the 2-tiered classification scheme for SILs (LSIL and HSIL), TBS 2001 also includes the equivocal categories of ASC-US and ASC-H. They represent equivocal LSIL and HSIL, respectively. However despite not being addressed in TBS, routine cervical smears may exhibit unequivocal LSIL in association with atypical cells cytomorphologically suspicious, but not sufficient to be interpreted as HSIL. Such cases are referred to as SILs of indeterminate grade in the TBS 2001 manual, but there was not a specific diagnostic category including the cervical smears which exhibit LSIL and ASC-H cytomorphology in the abbreviated versions of TBS $2001[10,11]$. There have been a number of studies focusing on this subset of cases, and many authors believe LSIL/ASC-H should be considered as a distinct diagnostic category [2$8]$ in TBS.

Many laboratories have identified this entity, and a number of terminologies have been coined to describe this type of abnormal Pap test $[4,5,9]$. Some of these terminologies are: (1) LSIL with rare atypical cells suggestive of but not diagnostic for HSIL, (2) LSIL with ASC-H, (3) LSIL cannot exclude HSIL, (4) LASC-H, (5) LSIL-H, and (6) LSIL/ASC-H $[1,2,4-7,12]$. In the Zeynep Kamil Hospital Pathology Laboratory, in which the current study was conducted, these lesions are defined as LSIL/ASC-H.
Many of these studies showed similar rates of CIN2/ CIN3 for LSIL/ASC-H, i.e. from 29 to $40.7 \%$ [1, 5-9, 12, 13], and a small number of studies showed higher rates of CIN2/CIN3 for LSIL/ASC-H, i.e. from 45 to $61 \%$ [2-4]. Our result was $43 \%$, which is consistent with the former results.

In all of these studies and the current study, the rates of LSIL/ASC-H for CIN2/CIN3 were similar to the rates of ASC-H for CIN2/CIN3, although they were slightly higher in some studies $[1,5]$ and slightly lower in others $[6,7,13]$. Our rate of ASC-H for CIN2/CIN3 was $41 \%$, which is slightly lower than our rate of LSIL/ASC-H for CIN2/CIN3. These results are very close to each other and do not show a significant statistical difference (table 2).

In spite of the similar rates of ASC-H and LSIL/ASC-H for CIN2/CIN3, both of these rates are higher than the diagnosis rate of LSIL (18\%) and lower than the diagnosis rate of HSIL (56\%) in our study. These results are consistent with previous publications $[1,3,5-7,9]$.

Because in all of these studies, including our own, LSIL/ASC-H had a high predictive value for HSIL and the risk of HSIL was similar to that of ASC-H, we believe LSIL/ASC-H should be classified as a distinct cytologic category. In line with these studies, our results support that the current clinical practice is valid (management is the same for ASC-H) $[3,4,6,9,13]$.

In the current study we also demonstrated that the CIN2 detection rate of LSIL/ASC-H (31\%) is higher than the CIN2 detection rate of ASC-H (10\%) and HSIL (16\%) and that these differences were statistically significant ( $p=0.003$ and $p=0.029$, respectively). Alsharif et al. [9] and Işık et al. [3] discussed these differences in their reports, yet other authors did not evaluated the number of CIN2 and CIN3 separately; instead they combined them in the HSIL group. Therefore, we cannot determine the actual CIN2 and CIN3 detection rates of LSIL/ASC-H 
from these nonseparated reports. Alsharif et al. [9] reported that the CIN2 detection rate of LSIL/ASC-H was $22.19 \%$, which was higher than the CIN3 detection rate of LSIL/ASC-H (10.95\%). Also, the CIN2 detection rate of LSIL/ASC-H was higher than the CIN2 detection rate of ASC-H (8.68\%), but slightly lower than that of HSIL (10.25\%). In the report of Işı et al. [3], the CIN2 detection rate of LSIL/ASC-H (30\%) was also higher than the CIN3 detection rate (5\%). The CIN2 detection rate of LSIL-ASC-H (30\%) was also the highest of all classifications (ASC-H 8\%, HSIL 13\%, and LSIL 0\%).

In spite of the different risks for cancer progression and spontaneous regression rates of CIN 2 and CIN3, they are grouped together as HSIL in TBS and are clinically managed in the same way $[10,14]$.

According to Mitchel et al. [15], 43\% of CIN2 lesions regress and $22 \%$ progress to carcinoma in situ. The respective rates for CIN3 lesions were 32 and $12 \%$ progression to carcinoma in situ [14].

More recently, Castle et al. [16] published estimates of the rate of spontaneous regression of biopsy-confirmed CIN2, finding that after 24 months of follow-up $43 \%$ of the lesions had regressed [14]. This is nearly identical to the meta-analytic study by Mitchel et al. [15].

On the other hand, while this entity was not rendered in different age groups, there may be differences in regression and progression rates in women of different age groups. McAllum et al. [17] reported that, under 25 years of age, the CIN2 regression rate is $62 \%$ [14]. In their study, 157 of 452 women under 25 years of age had been managed conservatively. Ninety-eight of 157 (62\%) women in this group showed spontaneous regression [15].

Since CIN2 has a higher regression rate compared to CIN3, especially in women under 25 years age [17], the CIN2 detection rate of LSIL/ASC-H may have more significance than we thought. There is a need for further investigation involving CIN2 regression rates in certain age groups to determine whether all women with HSIL (CIN2 and CIN3) should be managed in a similar way. If so, LSIL/ASC-H may be a stronger prognostic tool because of its higher detection rate of CIN2. The predictive ability of LSIL/ASC-H must be further evaluated with additional studies looking at the detection rates of CIN2 and CIN3 individually.

\section{References}

-1 Owens CL, Moats DR, Burroughs FH, Gustafson KS: 'Low-grade squamous intraepithelial lesion, cannot exclude high-grade squamous intraepithelial lesion' is a distinct cytologic category: histologic outcomes and HPV prevalence. Am J Clin Pathol 2007;128:398403.

-2 Kir G, Cetiner H, Gurbuz A, Karateke A: Reporting of 'LSIL with ASC-H' on cervicovaginal smears: is it a valid category to predict cases with HSIL follow-up? Eur J Gynecol Oncol 2004;25:462-464.

3 Kaygusuz EI, Cetiner H, Sahin D: LSIL/ASC$\mathrm{H}(\mathrm{LSIL}-\mathrm{H})$ in cervicovaginal smear: histopathological outcomes and clinical significance. Turk Patoloji Derg 2011;27:46-50.

4 Hunter C, Duggan MA, Duan Q, Power P, Gregoire J, Nation J: Cytology and outcome of LSIL: cannot exclude HSIL compared to ASCH. Cytopathology 2009;20:17-26.

$>5$ Sidham BS, Kumar N, Narayan R, Brotzman GL: Should LSIL with ASC-H (LSIL-H) in cervical smears be an independent category? A study on Surepath specimens with review of literature. Cytojournal 2007;4:7.

6 Difurio MJ, Mailhiot T, Sundborg MJ, Nauschuetz KK: Comparison of the clinical significance of the Papanicolaou test interpretations LSIL cannot rule out HSIL and ASCH. Diagn Cytopathol 2010, 38:313-317.
7 Elsheikh TM, Kirkpatrick JL, Wu HH: The significance of 'low-grade squamous intraepithelial lesion, cannot exclude high-grade squamous intraepithelial lesion' as a distinct squamous abnormality category in Papanicolaou tests. Cancer 2006,108:277-281.

-8 Finkelstein A, Bajor-Dattilo EB, Yang MC, Szeto O, Zhou F, Elgert P, Das K: The utility of 'low-grade intraepithelial lesion, cannot exclude high-grade' diagnosis: a single institution's experience. Acta Cytol 2012;56:383387.

$>9$ Alsharif M, Kjeldahl K, Curran C, Miller S, Gulbahce HE, Pambuccian SE: Clinical significance of the diagnosis of low-grade squamous intraepithelial lesion, cannot exclude high-grade squamous intraepithelial lesion. Cancer Cytopathol 2009;117:92-100.

10 Solomon D, Davey D, Kurman R, et al: The 2001 Bethesda System: terminology for reporting results of cervical cytology. JAMA 2002;287:2114-2119.

11 Solomon D, Nayar R: The Bethesda System for Reporting Cervical Cytology: Definition, Criteria and Explanatory Notes ed 2. New York, Springer, 2004.

12 Nasser SM, Cibas ES, Crum CP, Faquin WC: The significance of the Papanicolaou smear diagnosis of low-grade squamous intraepithelial lesion cannot exclude high-grade squamous intraepithelial lesion. Cancer Cytopathol 2003;99:272-276.
13 Power P, Gregoire J, Duggan M, Nation J: Low-grade Pap smears containing occasional high-grade cells as a predictor of high-grade dysplasia. J Obstet Gynaecol Can 2006;28: 884-887.

14 Wright TC, Ronnett BM, Kurman RJ, Ferency A: Precancerous lesions of the cervix; in Kurman RJ, Ellenson LH, Ronnett BM (eds): Blaustein's Pathology of the Female Genital Tract, ed 6. New York, Springer, 2011, pp 193-252.

15 Mitchell MF, Tortelero-Luna G, Wright T, Sarkar A, Richards-Kortum R, Hong WK, Schottenfeld D: Cervical human papillomavirus infection and intraepithelial neoplasia: a review. J Natl Cancer Inst Monogr 1996; 21:17-25.

16 Castle PE, Schiffman M, Wheeler CM, Solomon D: Evidence for frequent regression of cervical intraepithelial neoplasia-grade 2 . Obstet Gynecol 2009;113:18-25.

-17 McAllum B, Sykes PH, Sadler L, Macnab H, Simcock BJ, Mekhail AK: Is the treatment of CIN2 always necessary in women under 25 years old? Am J Obstet Gynecol 2011;205:478. e1-7. 